Submitted to Astrophys J.

\title{
Dynamics and neutrino signal of black hole formation in non-rotating failed supernovae. II. progenitor dependence
}

\author{
K. Sumiyoshi \\ Numazu College of Technology, Ooka 3600, Numazu, Shizuoka 410-8501, Japan \\ sumi@numazu-ct.ac.jp \\ S. Yamada \\ Science and Engineering 85 Advanced Research Institute for Science and Engineering, \\ Waseda University, Okubo, 3-4-1, Shinjuku, Tokyo 169-8555, Japan \\ and \\ H. Suzuki \\ Faculty of Science and Technology, Tokyo University of Science, \\ Yamazaki 2641, Noda, Chiba 278-8510, Japan
}

\begin{abstract}
We study the progenitor dependence of the black hole formation and its associated neutrino signals from the gravitational collapse of non-rotating massive stars, following the preceding study on the single progenitor model in Sumiyoshi et al. (2007). We aim to clarify whether the dynamical evolution toward the black hole formation occurs in the same manner for different progenitors and to examine whether the characteristic of neutrino bursts is general having the short duration and the rapidly increasing average energies. We perform the numerical simulations by general relativistic $\nu$-radiation hydrodynamics to follow the dynamical evolution from the collapse of pre-supernova models of $40 \mathrm{M}_{\odot}$ and $50 \mathrm{M}_{\odot}$ toward the black hole formation via contracting proto-neutron stars. For the three progenitor models studied in this paper, we found that the black hole formation occurs in $\sim 0.4-1.5 \mathrm{~s}$ after core bounce through the increase of proto-neutron star mass together with the short and energetic neutrino burst. We found that density profile of progenitor is important to determine the accretion rate onto the proto-neutron star and, therefore, the duration of neutrino
\end{abstract}


burst. We compare the neutrino bursts of black hole forming events from different progenitors and discuss whether we can probe clearly the progenitor and/or the dense matter.

Subject headings: supernovae: general — stars: neutron — black hole physics — neutrinos - hydrodynamics - equation of state

\section{Introduction}

The final fate of massive stars is one of key issues in stellar physics as well as astrophysics. The massive stars beyond $\sim 8 \mathrm{M}_{\odot}$ are the origin of compact objects, i.e. neutron stars and black holes, as the outcome of gravitational collapse at the end of their lives. They contribute also to the production of heavy elements through the explosive nucleosynthesis and to the high energy phenomena with the bursts of electro-magnetic radiations and neutrinos, which in turn play important roles in the evolution of galaxies. The massive stars in the wide range between $\sim 8 \mathrm{M}_{\odot}$ and $\sim 100 \mathrm{M}_{\odot}$ lead to various explosive phenomena such as core-collapse supernovae, hypernovae (or gamma ray bursts) and failed supernovae (Heger et al. 2003). Such energetic phenomena are dependent on the properties of progenitors such as masses, rotations and profiles, for example, as in the mass limit of ordinary supernovae and the branches of hypernovae and failed supernovae as a function of mass (Maeda \& Nomoto 2003). We focus here on the fate of non-rotating massive stars, as an extreme of failed supernovae, in the mass range of $\sim 40-50 \mathrm{M}_{\odot}$.

Such a death of massive stars attracts attention recently since it is one of possible channels of the black hole formation and is characterized by the unique neutrino signals (Liebendörfer et al. 2004; Sumivoshi et al. 2006, 2007): the short duration and increase of average energies and luminosities in time. This is in contrast to the ordinary supernova neutrinos, whose emissions from a nascent proto-neutron star last for $\sim 20 \mathrm{~s}$ with a gradual decrease of average energies and luminosities (Burrows 1988; Suzuki 1994). The black hole formation will be inevitable for massive stars beyond $\sim 40 \mathrm{M}_{\odot}$, which was estimated with large uncertainties to be the mass threshold for black hole formation by Fryer (1999). The dynamical collapse to a black hole is triggered by the intense accretion of matter onto the proto-neutron star. Note that this is different from the so-called delayed black hole formation of the proto-neutron star with a fixed mass (Keil \& Janka 1995; Pons et al. 1999; Baumgarte et al. 1996a) owing to the termination of accretion after a successful launch of shock wave. It should be also emphasized that the neutrino signal may be used as a probe into the equation of state of nuclear matter (Sumiyoshi et al. 2006) and exotic matter (Nakazato et al. 2008) in a different way from the delayed collapse of proto-neutron stars 
(Pons et al. 2001b. a).

This article is the second paper, following the first paper (Sumivoshi et al. 2007), of serial studies on the black hole formation associated with the short burst of energetic neutrinos. In the first paper, the evolution toward the black hole formation from the gravitational collapse of the $40 \mathrm{M}_{\odot}$ star have been examined by taking account of the dependence of equation of state. By solving general relativistic $\nu$-radiation hydrodynamics, they have followed the hydrodynamics up to the moment of black hole formation and the neutrino distributions during the whole evolution to obtain the detailed features of neutrino emission. They have shown that the black hole formation occurs in $\sim 1 \mathrm{~s}$ after the core bounce via proto-neutron star evolution, but it depends crucially on the equation of state of dense matter. The equation of state (EOS) controls the timing of the re-collapse from the proto-neutron star to the black hole since the EOS determines the maximum mass. The mass of proto-neutron star increases due to the accretion of material in the situation of failed shock wave and the dynamical collapse is triggered when the mass reaches the critical mass. We remark that the progenitor model of $40 \mathrm{M}_{\odot}$ by Woosley \& Weaver (1995) has been uniquely adopted in their studies and other studies on such massive stars (Liebendörfer et al. 2004).

In the first paper (Sumiyoshi et al. 2007), associated neutrino burst has been shown to be unique to identify the black hole formation and to probe the properties of dense matter. The neutrino burst is terminated by the black hole formation and the average energies of neutrinos increase toward the end because of high temperature realized in the collapsing proto-neutron stars. These features are again sensitive to the properties of equation of state, which determines the duration of neutrino burst and the properties of compressed matter at neutrino emitting region. The neutrino burst toward the black hole formation can, in principle, be detectable at the terrestrial neutrino detector facilities (Ikeda et al. 2007) and can be used as a signature of black hole formation and a probe of dense matter (Sumiyoshi et al. 2006). Moreover, if a collapse of massive star occurs in a nearby galaxy, the event may be observed, for example, in the proposed survey of disappearance of massive stars (Kochanek et al. 2008) and it will be possible to obtain the information on the progenitor. However, one has to provide the detailed features of neutrino burst by taking care of uncertainties of progenitor models in addition to dense matter if one wants to use them as templates for the neutrino search.

In this study, we make a first attempt to see whether the features of black hole formation reported in the first paper (Sumivoshi et al. 2007) are taken over to different progenitors. We adopt three stellar models as the initial condition of the numerical simulations to make the comparison with the evolutions by the numerical modeling we have adopted in the previous study (Sumiyoshi et al. 2007). In addition to the stellar model of $40 \mathrm{M}_{\odot}$ star by 
Wooslev \& Weaver (1995), we adopt the two stellar models by Hashimoto (1995) and by Tominaga et al. (2007). As a case of different mass, we adopt a $50 \mathrm{M}_{\odot}$ star by Tominaga et al. (2007), who studied the evolution of metal-poor massive stars. We have chosen the model with zero metallicity among their series of different metallicities. This model is the limiting case of metal-poor massive stars without mass loss. As a model for the same mass $\left(40 \mathrm{M}_{\odot}\right)$ but by a different method of stellar evolutionary calculation, we adopt a $40 \mathrm{M}_{\odot}$ star by Hashimoto (1995). The comparison between the different models enables us to explore the dependence of numerical results on the uncertainty in the stellar evolution.

The investigation on the progenitor dependence is important for the utilization of neutrino bursts as a probe of equation of state. If the neutrino burst is quite different depending on the progenitors, it becomes difficult to distinguish the differences found by the equation of state. Using the two sets of EOS (LS-EOS and Shen-EOS), it has been shown that the time duration of neutrino bursts differ by a factor of $\sim 2$ depending on the softness of EOS. If the progenitor models give large differences, they may smear out the EOS difference, which we want to use as a probe.

Our aim of the paper is, therefore, to make a first exploration to the dependence on the progenitors by adopting a small number of stellar models and to show that the dynamics toward the black hole formation is common in the massive stars having a large central iron core. We aim also to demonstrate that the neutrino signal has similar features once we set the equation of state. We analyze how the evolution is related with the density profile of progenitor, which determines the accretion rate. We discuss the characteristics of neutrino bursts obtained by different progenitors with the sets of equation of state for possible detection in future.

We plan this paper as follows. We briefly explain the method of numerical simulations in \$2, We compare the progenitors adopted for initial models and discuss the differences in $₫ 3$. We report the numerical results on the dynamics in $\$ \$ 4.1$ and 4.2 for two models through the comparison with the previous result. We describe the accretion rates and the profiles during the evolution in $\S \$ 4.3$ and 4.4 to explain the differences among the models. We discuss the characteristics of neutrino signals in the models to distinguish the progenitor and the equation of state in \$4.5. Implications of this work and summary will be made in $\S 95$ and 6 . 


\section{Numerical Simulations}

Numerical simulations are performed by the numerical code of general relativistic $\nu$ radiation hydrodynamics under the spherical symmetry (Yamada 1997; Yamada et al. 1999; Sumiyoshi et al. 2005). This numerical code has been applied to the gravitational collapse of massive stars to study the supernova explosion (Sumivoshi et al. 2005) and the black hole formation (Sumiyoshi et al. 2006, 2007; Nakazato et al. 2006, 2007). The detailed description on the numerical simulations of the gravitational collapse toward the black hole formation

can be found in Sumivoshi et al. (2007), which reported also the results for the progenitor of a $40 \mathrm{M}_{\odot}$ star by Woosley \& Weaver (1995). We adopt the same number of grids for lagrangian mass coordinate, variables in neutrino distributions for $\nu_{e}, \bar{\nu}_{e}, \nu_{\mu / \tau}$ and $\bar{\nu}_{\mu / \tau}$. The mesh for mass coordinate is optimized for each progenitor model to follow the accretion phase with an enough resolution using the rezoning method.

The fully implicit method of $\nu$-radiation hydrodynamics enables us to follow the long evolution over $\sim 1 \mathrm{~s}$ toward the black hole formation. The exact treatment of $\nu$-transfer together with hydrodynamics in general relativity permits the detailed evaluations of the neutrino fluxes from the contracting proto-neutron stars until the black hole formation. We define the black hole formation by finding the apparent horizon as explained in Nakazato et al. (2006) and Sumiyoshi et al. (2007).

We adopt the microphysics in the same manner as in Sumiyoshi et al. (2007) to facilitate the comparison of new results with the previous result for $40 \mathrm{M}_{\odot}$. We use the two sets of equation of state (EOS) for supernova simulations to assess the influence of EOS on different progenitors. The EOS by Shen et al. (1998a, b) (Shen-EOS), which is obtained by the relativistic mean field framework with the data of unstable nuclei, provides a rather stiff EOS having the maximum mass of neutron stars to be $2.2 \mathrm{M}_{\odot}$. The EOS by Lattimer \& Swesty (1991) (LS-EOS) is obtained by the extension of the compressible liquid model, taking a density dependence of energy based on the Skyrme-interaction. We have chosen the case of incompressibility of $180 \mathrm{MeV}$ among the three choices in LS-EOS as a representative of soft EOS having the maximum mass of neutron stars to be $1.8 \mathrm{M}_{\odot}$. The same set of weak interaction rates as in Sumivoshi et al. (2007) is adopted for the current simulations by implementing the standard formulation of Bruenn (1985) and the several extensions (Sumiyoshi et al. 2005). 


\section{Initial Models}

We adopt three different profiles from the pre-supernova models of massive stars as initial models. We have picked massive stars around $40 \mathrm{M}_{\odot}$, which are massive enough to have the black hole formation without the successful explosion. The choice of the progenitor models in the current study is meant for a first and small-scale trial exploration and does not reflect a possible great variety of massive stars. We use the central part of pre-supernova profiles up to $3 \mathrm{M}_{\odot}$ in baryon mass coordinate and set density, electron fraction and temperature distributions as initial configurations for numerical simulations.

As a base line model, we adopt a $40 \mathrm{M}_{\odot}$ star from the set of massive stars by Woosley \& Weaver (1995). This model has been used for the previous calculations of our studies of black hole formation and the details of numerical results can be found in Sumivoshi et al. (2007). We name this model W40 and will use as a reference to examine the new results. The size of iron core in $\mathrm{W} 40$ model is $1.98 \mathrm{M}_{\odot}$. As an example of other types of massive stars, we adopt a case of $50 \mathrm{M}_{\odot}$ with zero metallicity $(\mathrm{Z}=0)$ from the series of massive stars having low metallicities (Umeda \& Nomoto 2005; Tominaga et al. 2007). These zero-metal stars do not experience the mass loss during the stellar evolution and remain massive as in the initial stage (Umeda et al. 2000). This choice is meant to explore the dependence of the phenomena on the progenitor mass and the stellar evolution. The size of iron core is $1.88 \mathrm{M}_{\odot} \frac{1}{1}$ We name this model T50. In order to examine the difference due to the modeling of stellar evolutions for the same stellar mass, we adopt a $40 \mathrm{M}_{\odot}$ star by Hashimoto (1995). The size of iron core in this model is $1.88 \mathrm{M}_{\odot}$, which is largest among the series of his models up to $70 \mathrm{M}_{\odot}$. We name this model $\mathrm{H} 40$.

We compare the profiles of the three adopted models in Fig. 1. We can see quantitative differences among the three models though the profiles look similar at first glance. The density of T50 and H40 are higher than those of W40 in the central region whereas the order is reversed at the outer region. This difference in gradients of density profile from the center to the outer part is related to the difference in accretion rates onto proto-neutron star. Actually, the densities at $\mathrm{M}_{b}=2-3 \mathrm{M}_{\odot}$, which cover the range for the maximum mass of proto-neutron stars, are crucial to determine the final stage of accreting proto-neutron star through the free-fall time scale $(\sim 1 / \sqrt{G \rho})$.

The profile of electron fraction in T50 is slightly different from those in W40 and H40. There are several kinks at different positions due to the shell structure. The temperature of

\footnotetext{
${ }^{1}$ This value is determined by the criteria of $Y_{e}<0.50$, which corresponds roughly to the ${ }^{56} \mathrm{Ni}$ abundant region. A smaller value, $1.46 \mathrm{M}_{\odot}$, is obtained by the criteria of $Y_{e}<0.49$, which is the convention in their study (N. Tominaga, private communication, 2006).
} 
T50 and H40 are higher than that of W40 in the central region. This difference, however, smears out during the collapse when we compare the profiles at the timing with the same central density. We will discuss the profiles later in $\$ 4.4$ to explain the behavior of accretion rates.

\section{Numerical Results}

We present the numerical results for the cases of T50 (\$4.1) and H40 (\$4.2) through the comparison with the base line model of W40. We add a character, S or L for Shen-EOS or LS-EOS, respectively, in the model names to denote the choice of the EOS. We summarize the calculated models in Table 1. We discuss the differences among the three models and their implications in $\S \$ 4.34 .5$.

\section{1. $50 \mathrm{M}_{\odot}$ star by Tominaga et al.}

We show the radial trajectories of mass elements as a function of time after bounce for models with T50S and T50L in Figs. 2 and 3, respectively. The trajectories are plotted for each $0.02 \mathrm{M}_{\odot}$ in mass coordinate. Thick lines denote the trajectories for $0.5 \mathrm{M}_{\odot}, 1.0 \mathrm{M}_{\odot}$, $1.5 \mathrm{M}_{\odot}, 2.0 \mathrm{M}_{\odot}$ and $2.5 \mathrm{M}_{\odot}$. Thick dashed line represents the position of shock wave.

The general feature of evolution is found to similar to the case of corresponding W40 models. After the core bounce, the shock wave is launched up to $\sim 150 \mathrm{~km}$ in $0.1 \mathrm{~s}$ and then recedes gradually toward the surface of central object. The nascent proto-neutron star shrinks due to the mass increase slowly in T50S (quickly in T50L) by the intense accretion. The dynamical collapse occurs when the proto-neutron star mass exceeds a critical mass, which depends on the stiffness of EOS, and the black hole is formed.

The duration from the core bounce to the black hole formation is $1.51 \mathrm{~s}$ and $0.51 \mathrm{~s}$ for T50S and T50L, respectively, reflecting the difference between SH-EOS and LS-EOS. The duration in T50S is slightly longer than that in W40S, while the duration in T50L is slightly shorter than that in W40L. This is because the accretion rate is different between T50 and W40 in a time-dependent manner as we will see in $\$ 4.3$.

The duration of neutrino bursts is determined by the time toward the black hole formation. In Figs. 4 and 5 , we display the time evolution of average energies and luminosities of neutrinos emitted during the evolution described above. The corresponding results with the same EOS but using the progenitor of W40 are shown by thin lines in order to see the

progenitor dependence. In general, the features of neutrino emission for T50 resemble those 
for W40. The average energies for all species show the increasing tendency after bounce. The persistent increase for $\nu_{\mu / \tau}$ is remarkable among them. Looking more closely, small differences appear at late phase (after $\sim 0.4 \mathrm{~s}$ ). The luminosities for T50S become clearly lower than those for W40S at the late phase. This is related with a lower accretion rate in T50 than that in W40 at 1 s after bounce (see Fig. 8).

We remark that a rapid decrease of neutrino energies by the gravitational redshift that is expected at the terminal phase has not been observed yet in the current simulations. One has to follow the evolution further $(\lesssim 20 \mathrm{~ms})$ after the formation of the apparent horizon. For that purpose, however, a singularity avoiding scheme (Baumgarte et al. 1996b) should be implemented. We note also that some wiggles and/or jumps seen in Figs. 4 and 5 are numerical artifacts caused by insufficient numerical resolutions at the final phase of numerical simulations.

\section{2. $\quad 40 \mathrm{M}_{\odot}$ star by Hashimoto}

We display the radial trajectories of mass elements as a function of time after bounce for model with H40L in Fig. 6. The notation is the same as in Fig. 2. In this case, the evolution is much quicker than the cases in W40L and T50L. The time till the black hole formation is $0.36 \mathrm{~s}$ in H40L. Since the accretion rate in H40 is larger than those in W40 and T50, the mass of proto-neutron star reaches the critical mass earlier (see \$4.3). The contraction of central object is quick accordingly and, therefore, the density and temperature inside become high early on. It is recognizable that the position of shock wave behaves in a different manner from the other cases. The shock wave stays around $100 \mathrm{~km}$ after the initial launch instead of the recession. This is mainly because the neutrino luminosity is high due to the high accretion rate.

We show the time evolution of average energies and luminosities of neutrinos in Fig. 7 . The duration of neutrino burst is clearly shorter than that of W40L. The average energy of $\nu_{\mu / \tau}$ is larger and increases much faster than that of W40L, reflecting the different temperatures, whereas those of $\nu_{e}$ and $\bar{\nu}_{e}$ behave similarly. The neutrino luminosities of all species in H40L are higher than those in W40L. The luminosities of $\nu_{e}$ and $\bar{\nu}_{e}$ are proportional to the accretion luminosity $(\sim G M \dot{M} / r)$ and are high due to a high accretion rate $(\dot{M})$ and a more massive object $(M)$ at center. The high luminosity of $\nu_{\mu / \tau}$ originates from a high temperature in the massive object. The fact that the luminosity reflects the behavior of the accretion luminosity was also seen for the neutrino emissions at the shock breakout in the core-collapse of $11-20 \mathrm{M}_{\odot}$ stars (Thompson et al. 2003). The larger accretion rates found in the current study of $40-50 \mathrm{M}_{\odot}$ stars lead to more rapid increase of luminosity than for less 
massive stars.

\subsection{Accretion rate}

As we have discussed already, the accretion rate is a key factor to determine the behavior toward the black hole formation. In other words, the increasing rate of proto-neutron star mass is crucial to determine the growth time to the critical mass. We show in Fig. 8 the baryon mass of central object (corresponding to the proto-neutron star) as a function of time after bounce. We define here the central object, which is quasi-hydrostatic, as the one inside the position of shock wave. Note that the time derivative of the mass curve (i.e. the gradient of the curve) is the accretion rate. After the core bounce, the central object acquires $\sim 1.5 \mathrm{M}_{\odot}$ within $50 \mathrm{~ms}$. The proto-neutron star with a typical mass is formed immediately after the core bounce. This feature is common for all models of black hole forming collapse and is different from the case in ordinary supernovae, where the proto-neutron star $\left(\sim 1.5 \mathrm{M}_{\odot}\right)$ formation takes place in $\sim 0.3 \mathrm{~s}$ (Burrows 1988; Suzuki 1994). The mass increases gradually toward the critical mass in each model and the dynamical collapse to black hole occurs at the end point. The end point depends on the adopted progenitor and the equation of state.

Amazingly, the increase of mass in the cases of W40 and T50 are similar each other. Although the masses for T50 are slightly larger up to $0.6 \mathrm{~s}$ after bounce, their gradients (i.e. accretion rate) are the same, judging from the parallel curves between T50S and W40S (T50L and W40L). This similarity is the reason why the evolution of T50 and W40 and resulting neutrino signals are quite similar. Adopting LS-EOS, the black hole formation occurs around the same time $(\sim 0.5 \mathrm{~s})$ in the cases of T50L and W40L. In the case of SH-EOS, however, there is a difference of about $0.2 \mathrm{~s}$ between the cases of T50S and W40S. This is due to a smaller accretion rate in T50 at late phase ( $\gtrsim 0.6 \mathrm{~s}$ after bounce) than that in W40 as seen in the crossing of curves for T50S and W40S. We note that the small accretion rate at the late phase is related with the density profile of outer layer in the adopted progenitor.

It is remarkable that the behavior of H40L is different. The mass of central object for H40L increases faster than any other models. This means that the accretion rate is highest among the models and leads to the shortest neutrino burst. Since the mass becomes large soon after bounce, the density and temperature inside the proto-neutron star become high and they cause the quick rise of average energies and luminosities as discussed above. This

behavior is in accord with the analyses for their numerical results of the collapse of $40 \mathrm{M}_{\odot}$ star by Liebendörfer et al. (2004). 


\subsection{Profiles of density and temperature}

We examine the profiles of three models during the evolution to discuss the origin of differences in the black hole formation and the neutrino signals.

In order to clarify the origin of different histories of accretion rate, we make comparisons of density profiles for W40L, T50L and H40L at the time when the central density reaches $10^{11} \mathrm{~g} / \mathrm{cm}^{3}$ and at $30 \mathrm{~ms}$ after bounce in Fig. 9. In the upper panel of Fig. 9, we see the same profiles at center $\left(\mathrm{M}_{b} \lesssim 1 \mathrm{M}_{\odot}\right)$ when we compare at the same central density during the collapse, though we have seen the differences among the initial models as a whole in Fig. 1. This similarity of central cores implies the similar dynamics around core bounce. In fact, the density profiles of central part $\left(\mathrm{M}_{b} \lesssim 1.2 \mathrm{M}_{\odot}\right)$ at $30 \mathrm{~ms}$ after bounce are quite similar as seen in the lower panel of Fig. 9. We note that profiles of temperature and electron fraction are also similar in the central part.

It is remarkable to see the difference of densities in outer part $\left(M_{b} \gtrsim 1 M_{\odot}\right)$ among the three models. Since the density at each point determines the time scale of free fall $(\sim 1 / \sqrt{G \rho})$ onto the central object, the accretion rate $\left(\dot{M}=4 \pi r^{2} \rho v\right)$ is proportional to $\sim \rho^{3 / 2}$, therefore, the density difference is the origin of different accretion rates. In Fig. 9, the higher density in the outer profile of H40L informs that the accretion rate is higher up to the point at $\mathrm{M}_{b} \sim 2.4 \mathrm{M}_{\odot}$, where there is a crossing with the two other models. The profiles of T50L and W40L are rather similar each other though there are slight differences and a crossing at $\mathrm{M}_{b} \sim 2.1 \mathrm{M}_{\odot}$.

The difference in the densities of outer layers remains during the evolution of protoneutron star up to the black hole formation and leads to the different curves for proto-neutron star masses as we have seen in Fig. 8. At $30 \mathrm{~ms}$ after bounce, proto-neutron stars having $\sim 1.4 \mathrm{M}_{\odot}$ (in baryon mass) are formed and the rest of outer layers are accreting onto them in the free fall time. As they evolve, the baryon mass of proto-neutron star of H40L becomes larger than those of W40L and T50L because of the higher accretion rate. The cases of W40L and T50L in Fig. 8 are almost the same each other because of the similarity of density profiles. In the case of Shen-EOS, the cases of W40S and T50S in Fig. 8 behave similarly each other for the same reason. The increase in T50S at $\sim 1 \mathrm{~s}$ becomes slightly slower than that in W40S reflecting a crossing in the density profiles similar to the one discussed above.

In Figure 10, we compare the profiles of density and temperature at $300 \mathrm{~ms}$ after bounce for W40L, T50L and H40L. The central density for H40L is higher than those for W40L and T50L due to the larger baryon mass in proto-neutron star for H40L as seen in Fig. 8, The corresponding temperature for H40L is higher than others because of a more compact profile of proto-neutron star. We note that a kink at $\sim 100 \mathrm{~km}$ in the density profile of H40L 
corresponds to the shock position in accreting matter. The profiles of W40L and T50L are similar each other, though the profile in T50L is slightly more compact having higher density and temperature than those in W40L.

The compact profile in H40L leads to the higher average energies and luminosities in neutrino emission than those in the two other models. The higher average energy of $\nu_{\mu / \tau}$ in H40L arises from the higher temperature in H40L than others. The higher luminosities in H40L is also caused by the higher temperature and the compactness of central object. Since the accretion luminosities of $\nu_{e}$ and $\bar{\nu}_{e}$ are determined by the liberated energy in the gravitational potential of central proto-neutron star, a more massive and compact object leads to larger luminosities. In contrast, the neutrino energies and luminosities of W40L and T50L are similar each other because of the similar history of accretion and resulting evolution of proto-neutron stars. The same argument applies for the comparisons between W40S and T50S, in which the average energies are very close. The luminosities of T50S are somewhat smaller corresponding to the lower accretion rate at the late phase $\gtrsim 0.6 \mathrm{~s}$ after bounce.

To summarize, the density profile in progenitors determines the accretion rate in black hole forming collapse and affects the evolution of proto-neutron stars toward the black hole formation. The difference in proto-neutron star profiles in turn leads to the different features in neutrino emission. The characteristics of neutrino emission reflect the minute differences in density profiles of progenitors while the influence from the equation of state is more significant.

\subsection{Differences in neutrino signals}

Here we discuss the characteristics of neutrino bursts in the five calculated models aiming at discrimination of the effects from progenitors and EOSs. We summarize the numerical results regarding the black hole formation in Table 1 .

In order to probe the EOS, the time duration of neutrino burst is decisive. If the accretion rate of the progenitor can be determined, the measurement of the duration enables us to infer the critical mass of proto-neutron stars and to extract the stiffness of the EOS. In the current study, Shen-EOS is stiff and provides large critical masses of $\sim 2.7 \mathrm{M}_{\odot}$ in baryon mass $\left(\sim 2.3 \mathrm{M}_{\odot}\right.$ in gravitational mass, see Table 1$)$ for accreting proto-neutron stars. This large mass corresponds to the duration till the black hole formation over $1.3 \mathrm{~s}$ and is significantly longer than the cases of Lattimer-Swesty EOS as seen in Fig. 8, Since LattimerSwesty EOS is soft, the critical mass is $\sim 2.1 \mathrm{M}_{\odot}$ in baryon mass $\left(\sim 2.0 \mathrm{M}_{\odot}\right.$ in gravitational 
mass). The duration is $\sim 0.5 \mathrm{~s}$ with weak dependence on the progenitors among T50L, H40L and W40L. If one can get the information of the time duration accurately, one can infer the critical mass through the calculated curves of mass increase. For example, if we assume that the time duration of neutrino burst is $0.3 \mathrm{~s}$, the critical mass is in the range between $1.8 \mathrm{M}_{\odot}$ and $2.1 \mathrm{M}_{\odot}$ estimated from the range between $\mathrm{W} 40 \mathrm{~L}$ and $\mathrm{H} 40 \mathrm{~L}$ in Fig. 8. Therefore, one can estimate the critical mass with an error of $\sim 0.3 \mathrm{M}_{\odot}$ from the time duration within the uncertainty in the density profile of the progenitors. It is to be noted that the critical masses for proto-neutron stars, which contain abundant neutrinos at finite temperature, are different from the maximum masses for neutrino-less and cold neutron stars.

To reveal the differences due to progenitors, it is necessary to examine the detailed information of average energies and luminosities. When Shen-EOS is adopted, one has to wait for $\sim 1$ s to have enough differences between W40S and T50S. In case of LattimerSwesty EOS, it is rather difficult to distinguish the differences between the progenitors since the duration of burst is too short. The neutrino signals in W40L and T50L are too similar each other to discriminate. H40L is different from W40L and T50L, on the other hand, and may be distinguishable if the data on the average energy of $\nu_{\mu / \tau}$ and/or accurate luminosities are available.

In Figure 11, we compare the energy spectra of emitted neutrinos at $300 \mathrm{~ms}$ after bounce for H40L and W40L. The shapes of energy spectra for $\nu_{e}$ and $\bar{\nu}_{e}$ are similar between H40L and W40L, however, the peak values are higher for H40L. The peak position for $\nu_{\mu / \tau}$ for H40L is shifted for a higher energy, though the peak value is close to that for W40L. These differences appear as higher luminosities of all species and higher average energy of $\nu_{\mu / \tau}$ (but similar average energies for $\nu_{e}$ and $\bar{\nu}_{e}$ ) for H40L as seen at $300 \mathrm{~ms}$ in Fig:7. We compared the energy spectra at $300 \mathrm{~ms}$ after bounce also for T50L and W40L (not shown here). The energy spectra are similar each other except for slightly higher peak values for $\nu_{e}$ and $\bar{\nu}_{e}$ for T50L.

We remark here that the discussions on neutrino signals so far do not take into account the neutrino oscillations. One should take into account the changes of spectra during the propagation of neutrinos in outer layers. The observational aspects of the current numerical results considering the effects of neutrino oscillations are now under investigation and will be published elsewhere. 


\section{Discussions}

In this section, we discuss the perspective of the current work regarding the progenitor and the equation of state. We also remark on the observational chances of neutrino burst and implications to astrophysical phenomena with the black hole.

The present study on the black hole formation and the associated neutrino burst relies on the presupernova configurations of massive stars. Further studies with updated models of progenitors are definitely warranted. Necessary condition to have this type of phenomena is the appropriate density distribution to cause the intense accretion. In the three models (T50, H40 and W40) adopted in the current study, the size of iron core is large $\left(\sim 2 \mathrm{M}_{\odot}\right)$ and the dense region extends up to $\mathrm{M}_{b} \sim 3 \mathrm{M}_{\odot}$. This is different from the case of a $15 \mathrm{M}_{\odot}$ star by Woosley \& Weaver (1995), in which the iron core is smaller and the density slope is steep already at $\mathrm{M}_{b} \sim 1.5 \mathrm{M}_{\odot}$, for example. It is necessary to study further the density profiles of various progenitors and its relation to the accretion in order to examine whether the size of iron core is essential. It may be enough to have the flat profile of density so that the density remains high $\left(\sim 10^{6} \mathrm{~g} / \mathrm{cm}^{3}\right)$ at $\mathrm{M}_{b}=2-3 \mathrm{M}_{\odot}$, which corresponds to the critical mass of protoneutron stars. If the density profile is steep and the density is too low at the corresponding region in $40-50 \mathrm{M}_{\odot}$ stars, the black hole formation may take much longer $(\sim 10-100 \mathrm{~s})$ than the current case. Such events with longer neutrino bursts may look similar to the ordinary supernovae of $\sim 15 \mathrm{M}_{\odot}$ stars in terms of neutrino signals.

Regarding the uncertainty of progenitors, one should pay attention to the mass loss as well as the rotation. The progenitor models in Woosley \& Weaver (1995) and Hashimoto (1995) are obtained by the presupernova evolution without the mass loss. The inclusion of the mass loss leads to the smaller mass at the precollapse stage even from the large initial mass $\left(\right.$ ex. $\left.50 \mathrm{M}_{\odot}\right)$ at the main sequence. The iron core mass and the density distribution may end up to be similar to those in the progenitors of about $20 \mathrm{M}_{\odot}$. In this regard, metal poor massive stars are more likely to be the progenitors for the black hole formation as studied in the current work. In fact, T50 adopted in the current study is the one with zero metallicity that forbids the mass loss. Its large central core results in the necessary intense accretion, which in turn leads to the quick black hole formation with short neutrino bursts. Whether the change of evolution toward the solar metallicity may cause the suppression of the current scenario remains to be seen along with the systematic studies on the metallicity dependence of progenitors and the careful studies on the mass loss.

We study the black hole formation under the spherical symmetry. This geometrical assumption limits ourselves to the non-rotating massive stars among varieties in rotation rates. One should note, however, that the spherical symmetry is a good approximation if the rotation is not significantly fast. It is challenging to study the collapse of rapidly rotating 
massive stars, which are possible progenitors for hypernovae and gamma ray bursts. One has to perform the fully general relativistic calculations in multi-dimensions to follow the evolution toward the black hole formation. Such challenges are made in the numerical simulations of general relativistic hydrodynamics but with simple treatment of neutrino transfer, microphysics (ex. EOS) and progenitors (Shibata \& Sekiguchi 2005; Sekiguchi \& Shibata 2005, 2007). Multi-dimensional simulations with proper treatment of neutrino transfer are awaited for to predict the features of neutrino emission.

The rotating collapse to the black hole and the following evolution is also interesting from the aspect of nucleosynthesis. In the context of collapsar scenario, the ejection of neutronrich material in the jet from the surroundings of black hole and the neutrino-driven winds from the accretion disk around the black hole are proposed to be the possible site for the heavy elements production including r-process elements (Surman et al. 2006; Fujimoto et al. 2007). Although we performed the numerical simulations in the limit of no rotation, one may use the current results to guess the time duration till the black hole formation and the accretion rate of material, which are influential in the scenario of nucleosynthesis. The time duration obtained in the current work is a lower limit since the maximum mass of rotating proto-neutron stars is larger than the non-rotating ones (Sumiyoshi et al. 1999) and the rotation supports accreting material away due to the centrifugal force. The accretion rate in the current study is an upper limit, accordingly. Large accretion rates in the disk formed around black hole, which are assumed in the studies of nucleosynthesis of r-process (Surman et al. 2006), may be limited from the density profile of progenitors as we have pointed out.

The observational possibility to detect the neutrino bursts toward the black hole formation from massive stars deserves to be mentioned. Occurrence rate of the phenomena (non-rotating black hole formation and its short neutrino burst) as in the current scenario is proportional to the fraction of massive stars having large masses for intense accretion without significant rotation. This depends on the initial mass function and rotation rates with large uncertainties, but such stars comprise a certain fraction $(\sim 30 \%)$ of massive stars, which can be estimated from the Salpeter's initial mass function with an assumption on the mass threshold $\left(\sim 25 \mathrm{M}_{\odot}\right)$ for the black hole formation. Kochanek et al. (2008) estimated from the observations done so far that the rate of black hole formation could be comparable to the rate of normal core-collapse supernovae. Observational analyses of explosion energies and $\mathrm{Ni}$ productions for various supernovae indicate that there have been observed at least several faint supernovae (SN 1997D and SN 1999br, for example) from massive stars beyond $20 \mathrm{M}_{\odot}$ without significant rotations, which may be akin to the event considered in this paper (Nomoto et al. 2007). 
The planned survey of disappearance of massive stars within a distance of $10 \mathrm{Mpc}$ (Kochanek et al. 2008) will find both dim supernovae and collapses with no optical display that lead to the black hole formation. If they occur in the Galaxy or nearby galaxies, the information on progenitor will be obtained by the optical records before it disappeared. In addition, thousands of neutrinos will be detected at the terrestrial facilities. Neutrino data combined with optical ones will provide us with the information on the dynamics of black hole formation and constrain the properties of dense matter and the progenitor. In the search of supernova neutrinos by the Super-Kamiokande detector, neither neutrinos associated with black hole formation nor ordinary supernova neutrinos are detected during the period of their survey unfortunately (Ikeda et al. 2007).

The numerical result on the massive star with zero metallicity (T50) suggests that the black hole formation in the current scenario may occur for Pop III stars in the early Universe (see also Nakazato et al. (2006, 2007)). Since the massive stars become more popular in the metal poor epoch, the neutrinos from black hole formation in the early stage of galaxies may contribute to the relic signal as back ground neutrinos. Although the signal is short, higher energies and luminosities may help to enhance this contribution and they may not be negligible. This argument again depends on the fraction of massive stars resulting the current scenario through the evolution of galaxies.

Before we close the discussion, we comment on the choice of equation of state. Although density and temperature become extremely high enough to have new degrees of freedom such as hyperons and quarks in the evolution toward the black hole formation, we adopt the two sets of EOS within the nucleonic degree of freedom in the current study to assess the progenitor dependence. Studies on the modifications of the evolution of black hole formation and the neutrino signal due to hyperon mixtures and the appearance of quark phase are now under way and will be reported in separate papers (Nakazato et al. 2008).

\section{Summary}

We study the black hole formation and the associated neutrino emission from the gravitational collapse of massive stars to clarify the dependence on the progenitor models following the basic study of the phenomena in the first paper (Sumiyoshi et al. 2007) of the series. We perform the sets of numerical simulations of general relativistic $\nu$-radiation hydrodynamics under the spherical symmetry to follow the collapse of massive stars of $40 \mathrm{M}_{\odot}$ and $50 \mathrm{M}_{\odot}$ adopting different models of the presupernova evolution. We clarify that the characteristics of evolution of proto-neutron stars toward the black hole formation and the associated short burst of neutrinos are found similar in the numerical simulations for the three progenitor 
models.

We find that the different progenitor models may result in different rates of matter accretion onto the proto-neutron star born in the collapse of massive stars. The different accretion rates can lead to different histories of increasing mass of proto-neutron stars toward the critical mass and to different durations till the black hole formation. In order to extract the properties of equation of state from the neutrino burst toward the black hole formation from massive stars, as proposed in the previous studies (Sumivoshi et al. 2006, 2007), it is necessary to constrain the uncertainty from progenitor models. In the comparison of the three progenitors we studied, the change of time duration is found not so large as the difference originating from the two sets of equation of state. The change of time profiles of average energies and luminosities of neutrinos due to progenitors is found to be minor as well. Note, however, that the present study is a first trial exploration and more systematic investigations are obviously necessary to see if our conclusion is generic or not.

We demonstrate that there is a relation between the density profile of progenitor and the accretion history to cause the difference in black hole formation. A higher density at $\mathrm{M}_{b}=2.0_{-}$ $3.0 \mathrm{M}_{\odot}$ in a progenitor results in a shorter free fall time and a higher accretion rate. A flat density profile of core in massive stars is a key factor to cause the rapid formation of black hole formation in $\sim 1 \mathrm{~s}$ together with the characteristic neutrino burst. When the density gradients are different as in the comparison between W40L and H40L, the density profiles may be distinguished from the detailed information of neutrino detections. This relation of the density profile of progenitors to the accretion rates is helpful to discuss the evolution after the collapse of massive stars in other stellar models and may be useful to infer the accretion dynamics of rotating collapse to the black hole formation and associated explosive phenomena such as collapsars. Further studies on rotating collapse of massive stars with intense accretion are called for to clarify whether the outcome may lead to possible success of modeling gamma ray burst and/or nucleosynthesis of heavy elements.

The authors are grateful to H. Shen, K. Oyamatsu, A. Ohnishi, C. Ishizuka and H. Toki for the collaborations on the tables of equation of state for supernova simulations. We thank K. Nakazato, S. Fujimoto, K. Kotake, M. Liebendorfer and T. Kajino for fruitful discussions and keen comments. We thank N. Tominaga, H. Umeda, K. Nomoto and M. Hashimoto for providing their progenitor models and further correspondence. K. S. expresses his gratitude to W. Hillebrandt and T.-H. Janka for the hospitality at Max Planck Institute für Astrophysik where a part of this work was done. The numerical simulations were performed on VPP5000 at Center for Computational Astrophysics, CfCA, of National Astronomical Observatory of Japan (wks06a, iks13a, uks06a), on Altix3700Bx2 at the Tokai branch of Japan Atomic Energy Agency (JAEA), on Altix3700Bx2 at YITP in Kyoto University and SX at 
RCNP, Osaka University. K. S. is grateful to T. Maruyama and S. Chiba for the arrangement of computing resources at JAEA. This work is partially supported by the Grants-in-Aid for the Scientific Research (18540291, 18540295, 19104006, 19540252) of the MEXT of Japan, Academic Frontier Project of the MEXT, KEK LSSP (07-05) and the 21st-Century COE Program "Holistic Research and Education Center for Physics of Self-organization Systems".

\section{REFERENCES}

Baumgarte, T. W., Janka, H.-T., Keil, W., Shapiro, S. L., \& Teukolsky, S. A. 1996a, Astrophys. J., 468, 823

Baumgarte, T. W., Shapiro, S. L., \& Teukolsky, S. A. 1996b, Astrophys. J., 458, 680

Bruenn, S. W. 1985, Astrophys. J. Suppl., 58, 771

Burrows, A. 1988, Astrophys. J., 334, 891

Fryer, C. 1999, Astrophys. J., 522, 413

Fujimoto, S., Hashimoto, M., Kotake, K., \& Yamada, S. 2007, Astrophys. J., 656, 382

Hashimoto, M. 1995, Prog. Theor. Phys., 94, 663

Heger, A., Fryer, C., Woosley, S., Langer, N., \& Hartmann, D. 2003, Astrophys. J., 591, 288

Ikeda, M. et al. 2007, Astrophys. J., 669, 519

Keil, W. \& Janka, H.-T. 1995, Astron. Astrophys., 296, 145

Kochanek, C. S., Beacom, J. F., Kistler, M. D., Prieto, J. L., Stanek, K. Z., Thompson, T. A., \& Yuksel, H. 2008, Astrophys. J., in press, arXiv:0802.0456

Lattimer, J. M. \& Swesty, F. D. 1991, Nucl. Phys., A535, 331

Liebendörfer, M., Messer, O. E. B., Mezzacappa, A., Bruenn, S. W., Cardall, C. Y., \& Thielemann, F.-K. 2004, Astrophys. J. Suppl., 150, 263

Maeda, K. \& Nomoto, K. 2003, Astrophys. J., 598, 1163

Nakazato, K., Sumiyoshi, K., \& Yamada, S. 2006, Astrophys. J., 645, 519

—. 2007, Astrophys. J., 666, 1140

—. 2008, Phys. Rev., D77, 103006 
Nomoto, K., Tominaga, N., Tanaka, M., Maeda, K., \& Umeda, H. 2007, in Supernova 1987A: 20 Years After: Supernovae and Gamma-Ray Bursters, ed. S. Immler, K. Weiler, \& R. McCray (American Institute of Physics), arXiv:0707.2187

Pons, J. A., Miralles, J. A., Prakash, M., \& Lattimer, J. M. 2001a, Astrophys. J., 553, 382

Pons, J. A., Reddy, S., Prakash, M., Lattimer, J. M., \& Miralles, J. A. 1999, Astrophys. J., 513,780

Pons, J. A., Steiner, A. W., Prakash, M., \& Lattimer, J. M. 2001b, Phys. Rev. Lett., 86, 5223

Sekiguchi, Y. \& Shibata, M. 2005, Phys. Rev., D71, 084013

—. 2007, Prog. Thoer. Phys., 117, 1029

Shen, H., Toki, H., Oyamatsu, K., \& Sumiyoshi, K. 1998a, Nucl. Phys., A637, 435

—. 1998b, Prog. Thoer. Phys., 100, 1013

Shibata, M. \& Sekiguchi, Y. 2005, Phys. Rev., D71, 024014

Sumiyoshi, K., Ibanez, J. M., \& Romero, J. V. 1999, Astrophys. J. Suppl., 134, 39

Sumiyoshi, K., Yamada, S., \& Suzuki, H. 2007, Astrophys. J., 667, 382

Sumiyoshi, K., Yamada, S., Suzuki, H., \& Chiba, S. 2006, Phys. Rev. Lett., 97, 091101

Sumiyoshi, K., Yamada, S., Suzuki, H., Shen, H., Chiba, S., \& Toki, H. 2005, Astrophys. J., 629,922

Surman, R., McLaughlin, G. C., \& Hix, W. R. 2006, Astrophys. J., 643, 1057

Suzuki, H. 1994, in Physics and Astrophysics of Neutrinos, ed. M. Fukugita \& A. Suzuki (Tokyo: Springer-Verlag), 763

Thompson, T. A., Burrows, A., \& Pinto, P. 2003, Astrophys. J., 539, 865

Tominaga, N., Umeda, H., \& Nomoto, K. 2007, Astrophys. J. Suppl., 660, 516

Umeda, H. \& Nomoto, K. 2005, Astrophys. J., 619, 427

Umeda, H., Nomoto, K., \& Nakamura, T. 2000, in Proceedings of the First Stars, ed. A. Weiss, T. Abel, \& V. Hill (Berlin: Springer), 150, astro-ph/9912248 
Woosley, S. E. \& Weaver, T. 1995, Astrophys. J. Suppl., 101, 181

Yamada, S. 1997, Astrophys. J., 475, 720

Yamada, S., Janka, H.-T., \& Suzuki, H. 1999, Astron. Astrophys., 344, 533 
Table 1: Summary of calculated models

\begin{tabular}{cccccccc}
\hline \hline Model & Progenitor & $\mathrm{M}_{\text {prog }}$ & $\mathrm{M}_{F e}$ & EOS & $\mathrm{M}_{b}^{\max }\left[\mathrm{M}_{\odot}\right]$ & $\mathrm{M}_{g}^{\max }\left[\mathrm{M}_{\odot}\right]$ & $\mathrm{t}_{B H}[\mathrm{~s}]$ \\
\hline W40S & WW95 $^{\mathrm{a}}$ & 40 & 1.98 & Shen & 2.66 & 2.38 & 1.35 \\
W40L & WW95 $^{4}$ & 40 & 1.98 & LS & 2.10 & 1.99 & 0.57 \\
T50S & TUN07 $^{\mathrm{b}}$ & 50 & 1.88 & Shen & 2.65 & 2.33 & 1.51 \\
T50L & TUN07 $^{50}$ & 1.88 & LS & 2.11 & 2.01 & 0.51 \\
H40L & H95 $^{\mathrm{c}}$ & 40 & 1.88 & LS & 2.17 & 2.08 & 0.36 \\
\hline
\end{tabular}

${ }^{a}$ WW95: Woosley \& Weaver (1995)

${ }^{b}$ TUN07: Umeda \& Nomoto (2005); Tominaga et al. (2007)

${ }^{c}$ H95: Hashimoto (1995)

Note. - $\mathrm{M}_{\text {prog }}$ and $\mathrm{M}_{F e}$ is the mass of progenitor and the iron core mass, respectively. $\mathrm{M}_{b}^{\max }$ and $\mathrm{M}_{g}^{\max }$ are the masses of proto-neutron star just prior to the re-collapse in baryon and gravitational masses, respectively. $\mathrm{t}_{B H}$ is the time at the black hole formation measured from the core bounce. See the main text for details. 


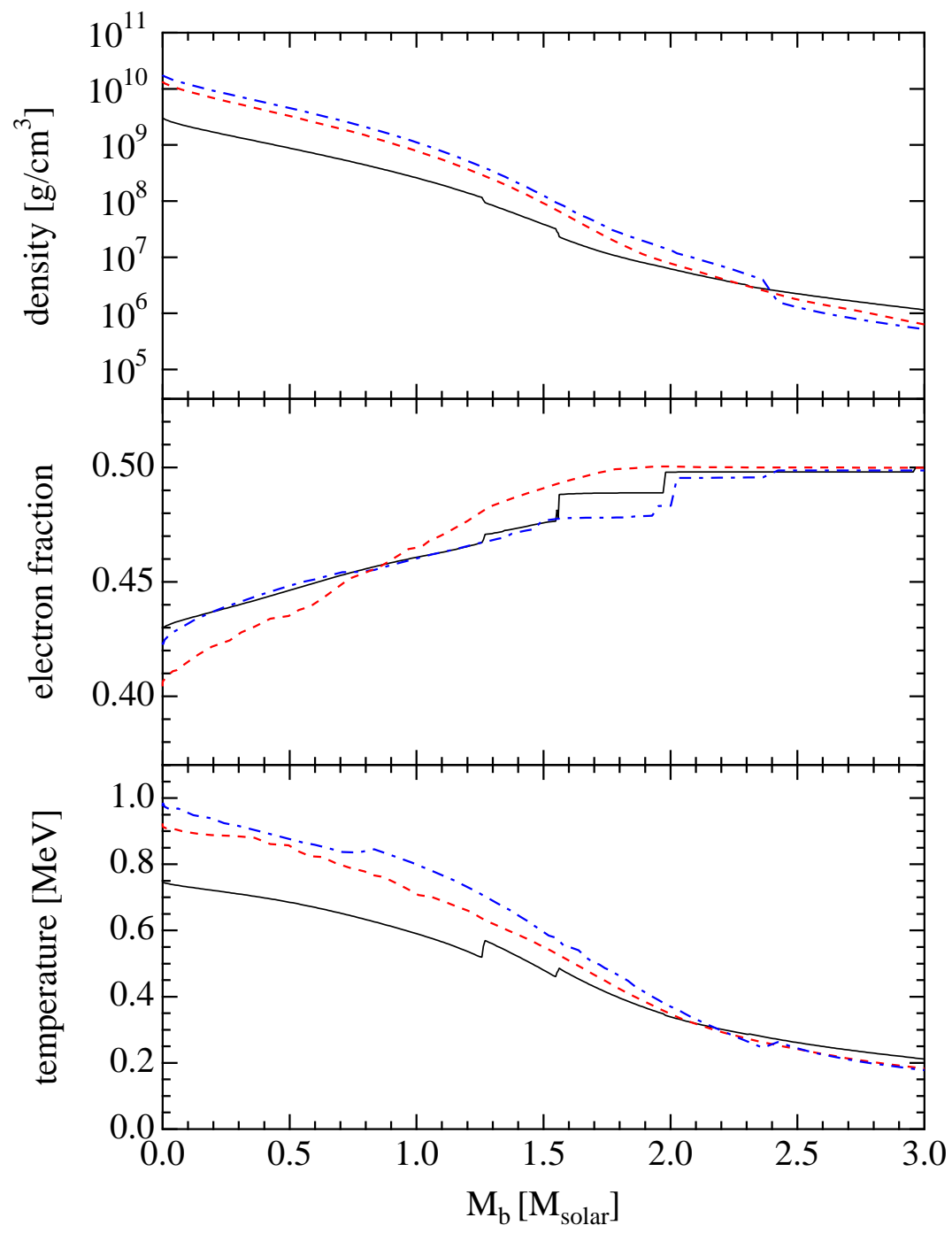

Fig. 1.- Density, electron fraction and temperature distributions of progenitor models are shown as a function of baryon mass coordinate by solid, dashed and dash-dotted lines for W40, T50 and H40, respectively. 


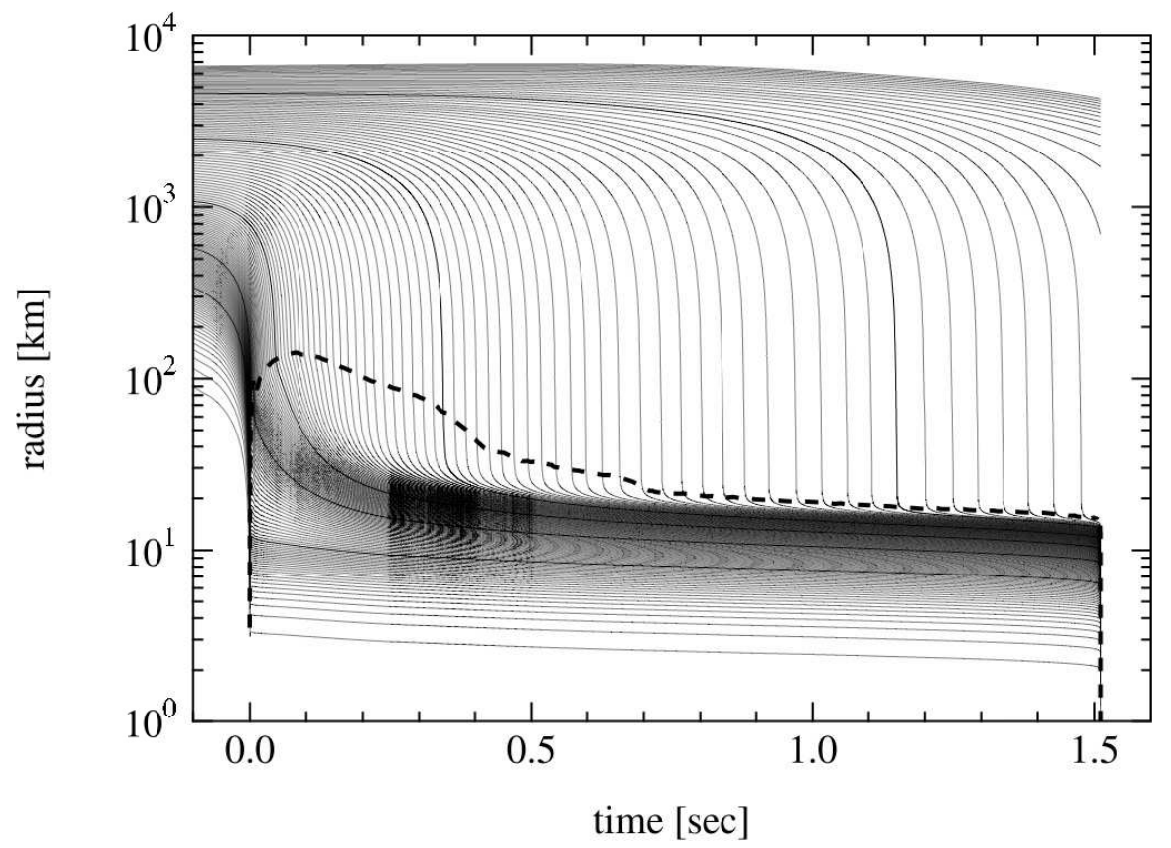

Fig. 2.- Radial trajectories of mass elements of the core of $50 \mathrm{M}_{\odot}$ star as a function of time after bounce in model T50S. The location of shock wave is displayed by a thick dashed line.

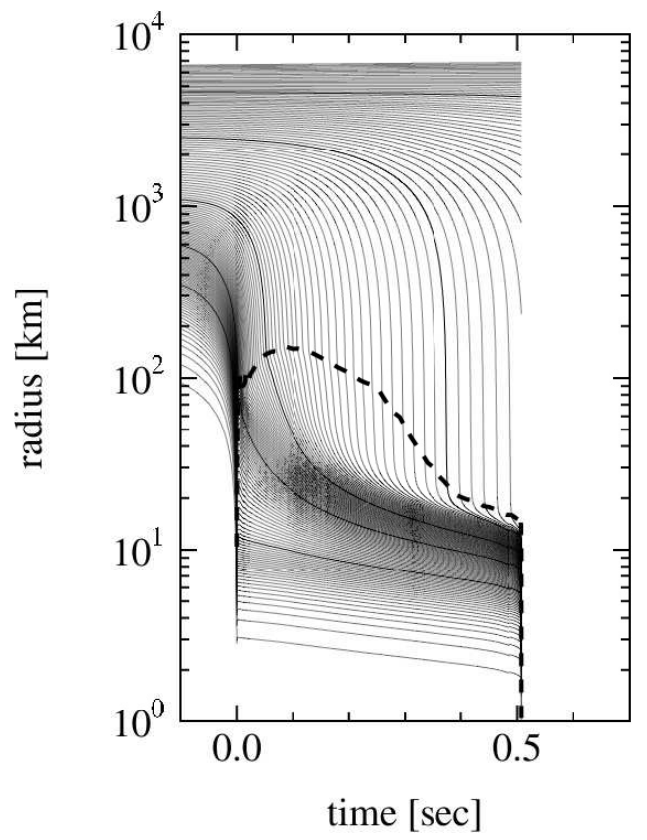

Fig. 3.- Radial trajectories of mass elements of the core of $50 \mathrm{M}_{\odot}$ star as a function of time after bounce in model T50L. The location of shock wave is displayed by a thick dashed line. 


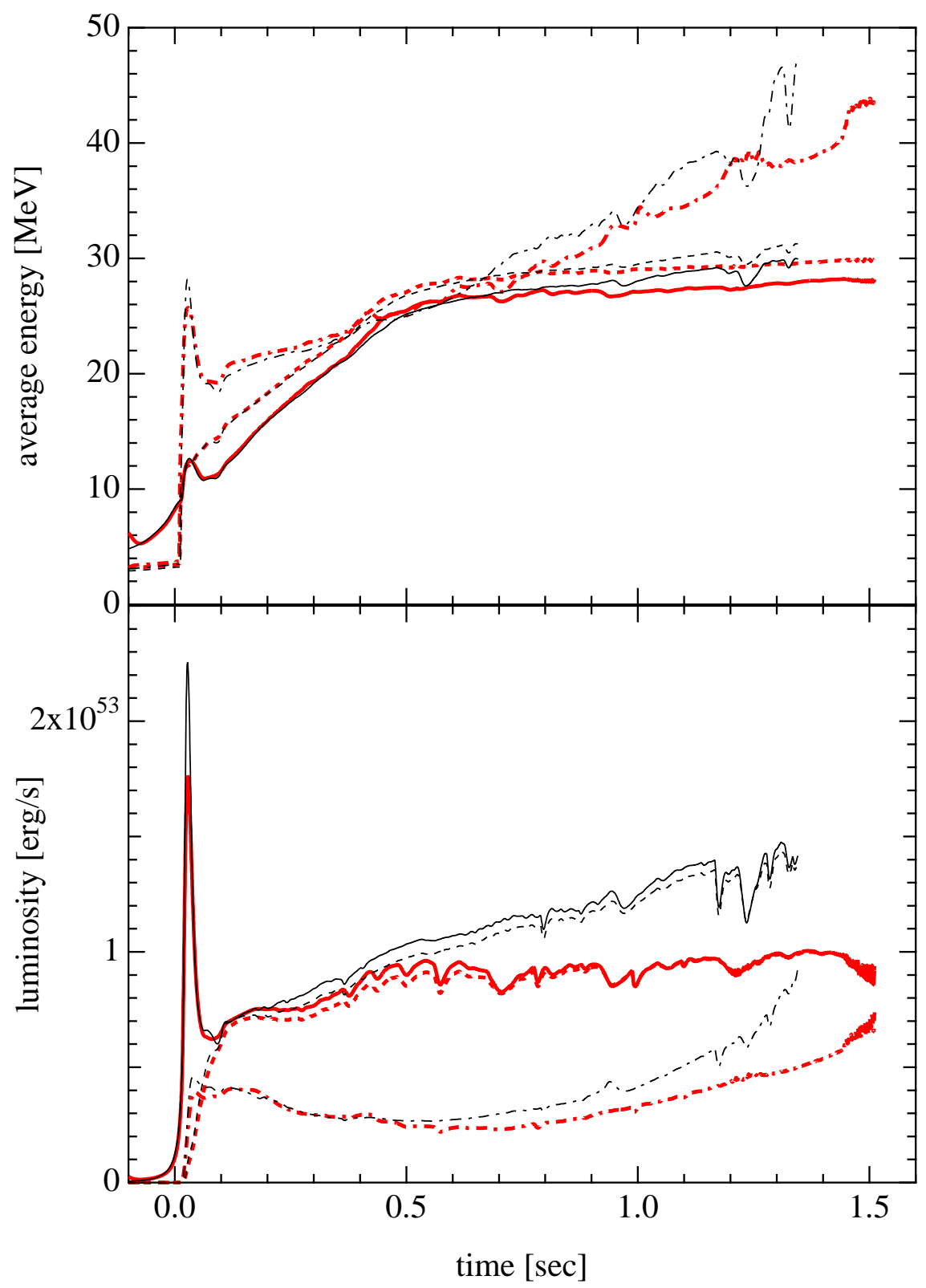

Fig. 4.- Average energies and luminosities of $\nu_{e}$ (solid), $\bar{\nu}_{e}$ (dashed) and $\nu_{\mu / \tau}$ (dash-dotted) for model T50S are shown as a function of time after bounce. The results for model W40S are shown by thin lines. 


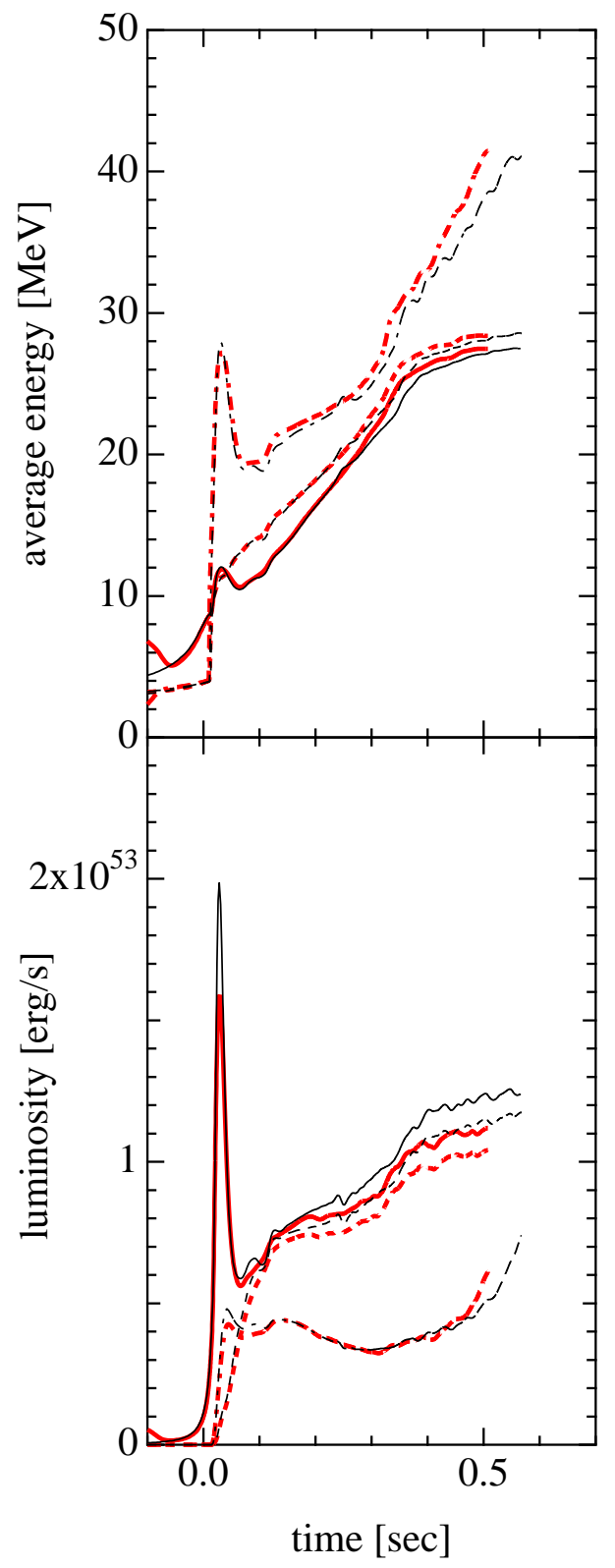

Fig. 5.- Average energies and luminosities of $\nu_{e}$ (solid), $\bar{\nu}_{e}$ (dashed) and $\nu_{\mu / \tau}$ (dash-dotted) for model T50L are shown as a function of time after bounce. The results for model W40L are shown by thin lines. 


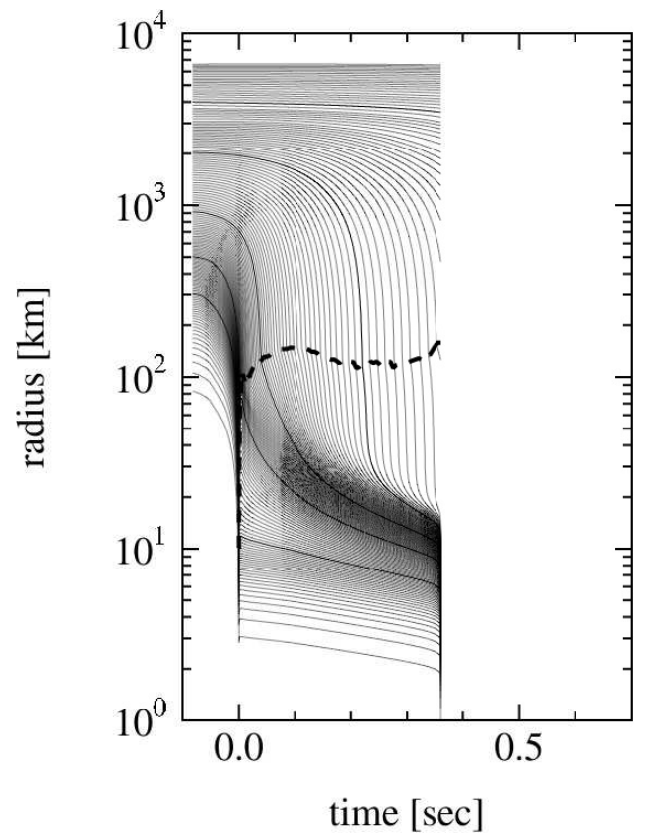

Fig. 6.- Radial trajectories of mass elements of the core of $40 \mathrm{M}_{\odot}$ star as a function of time after bounce in model H40L. The location of shock wave is displayed by a thick dashed line. 


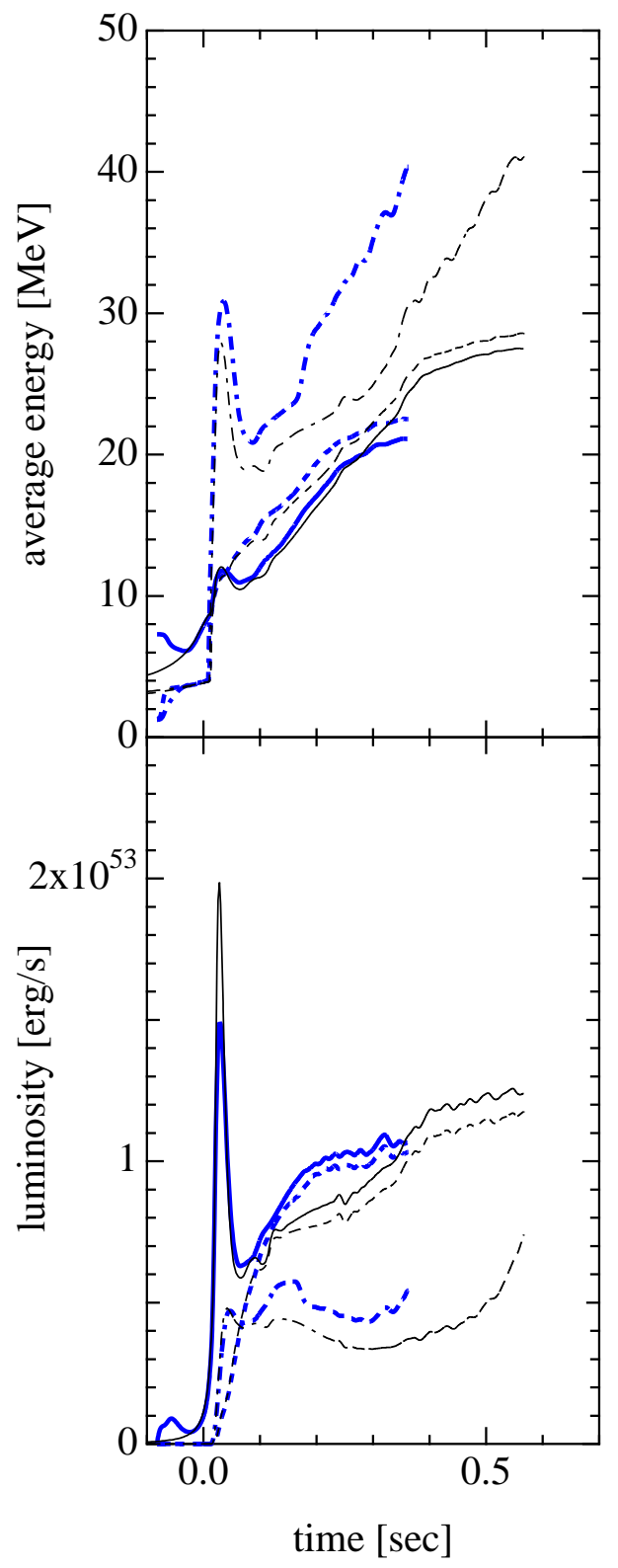

Fig. 7.- Average energies and luminosities of $\nu_{e}$ (solid), $\bar{\nu}_{e}$ (dashed) and $\nu_{\mu / \tau}$ (dash-dotted) for model H40L are shown as a function of time after bounce. The results for model W40L are shown by thin lines. 


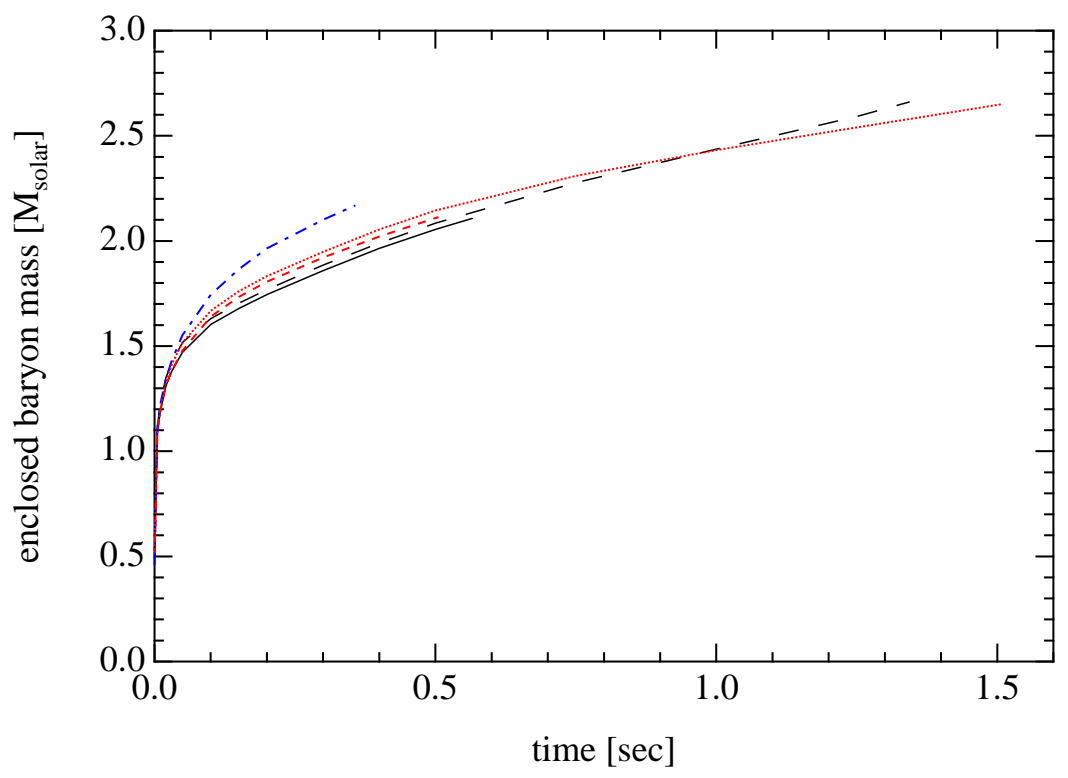

Fig. 8.- Enclosed baryon mass in the central object (proto-neutron star) as a function of time after bounce for models W40S (long-dashed), W40L (solid), T50S (dotted), T50L (dashed) and H40L (dash-dotted). 

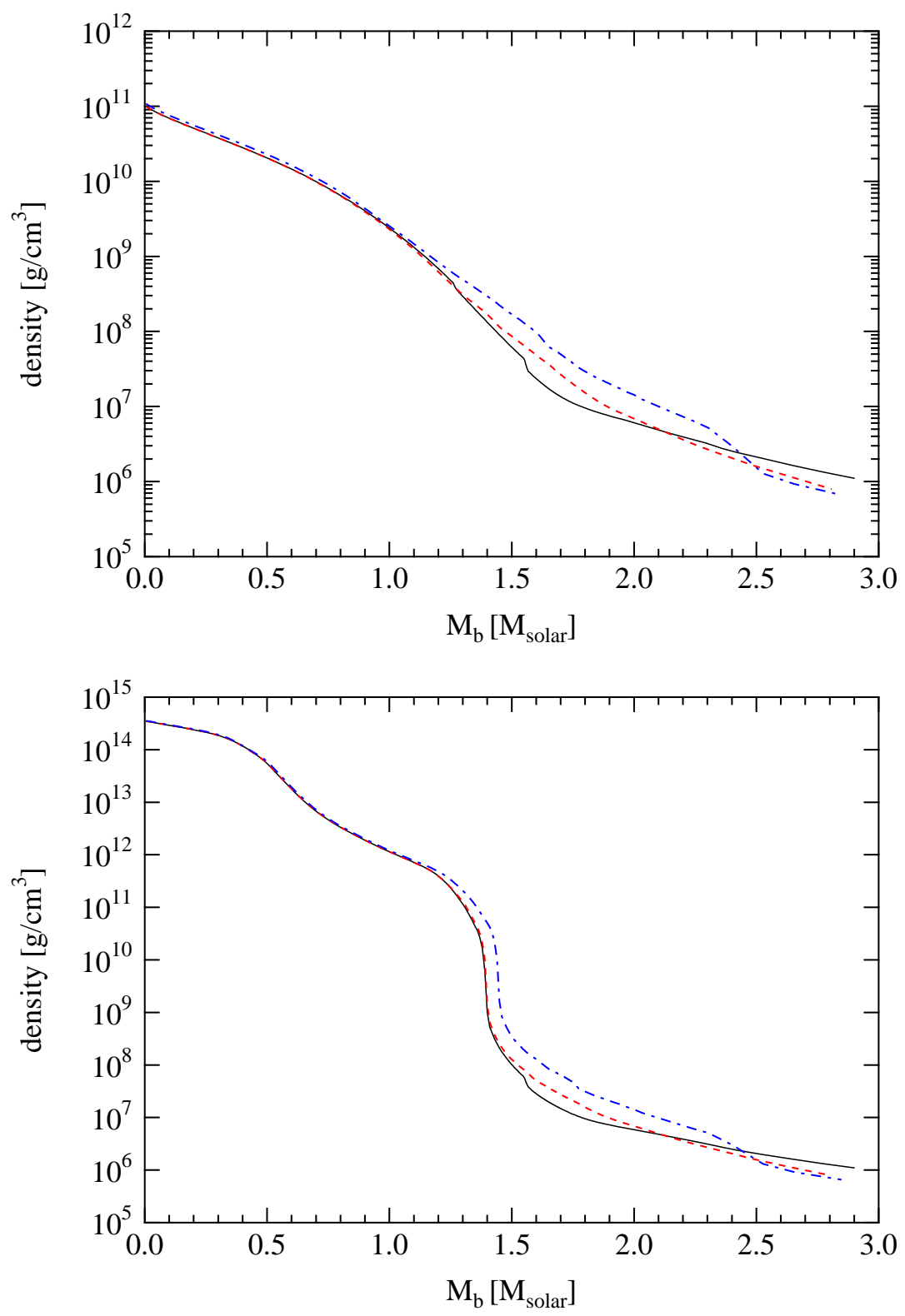

Fig. 9.- Density distributions for W40L, T50L and H40L are shown as a function of baryon mass coordinate by solid, dashed and dash-dotted lines, respectively. Upper and lower panels show the profiles at the time when the central density reaches $10^{11} \mathrm{~g} / \mathrm{cm}^{3}$ and at $30 \mathrm{~ms}$ after bounce, respectively. 


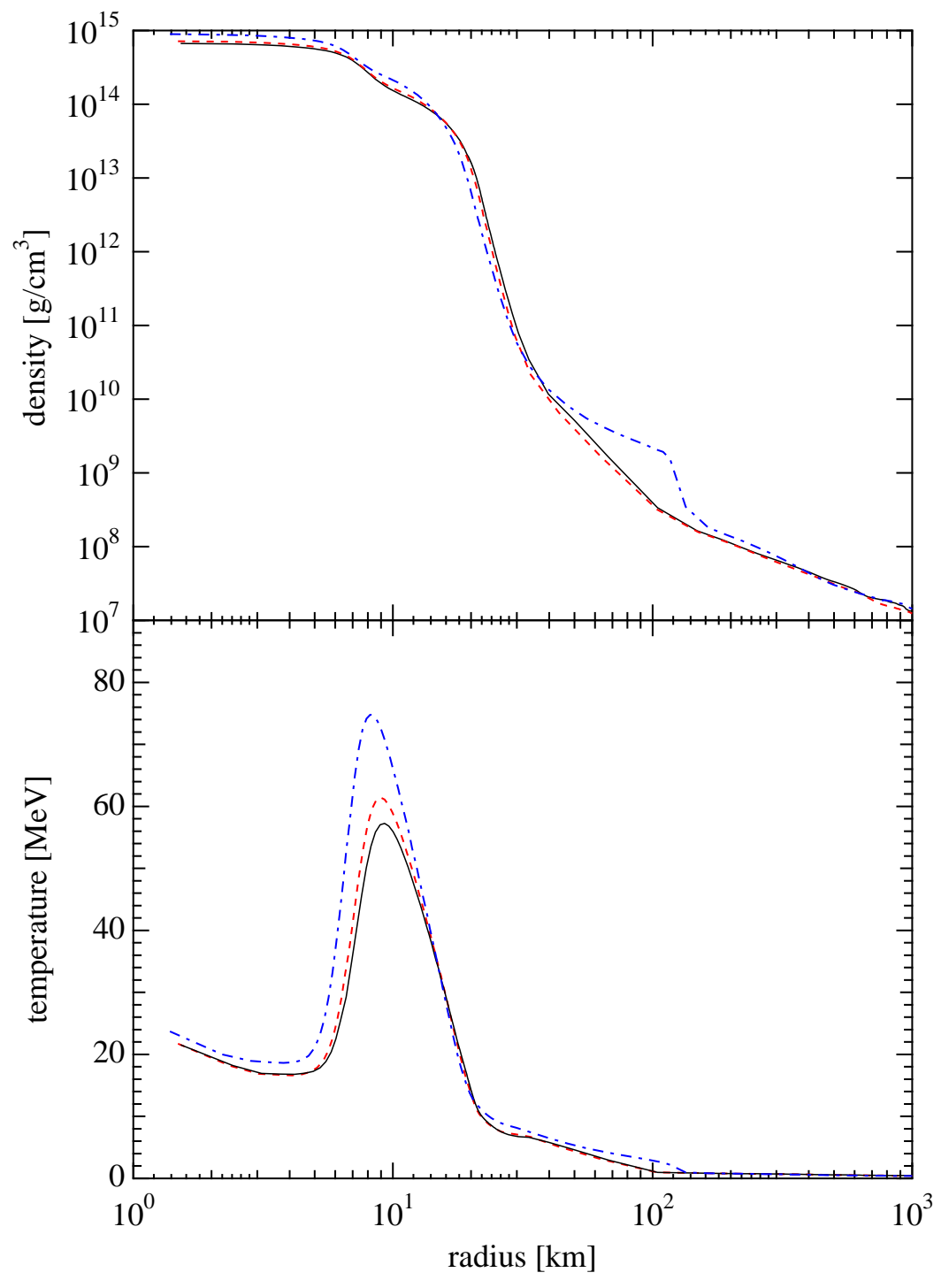

Fig. 10.- Density (upper panel) and temperature (lower panel) distributions for W40L, T50L and H40L at $300 \mathrm{~ms}$ after bounce are shown as a function of radius by solid, dashed and dash-dotted lines, respectively. 


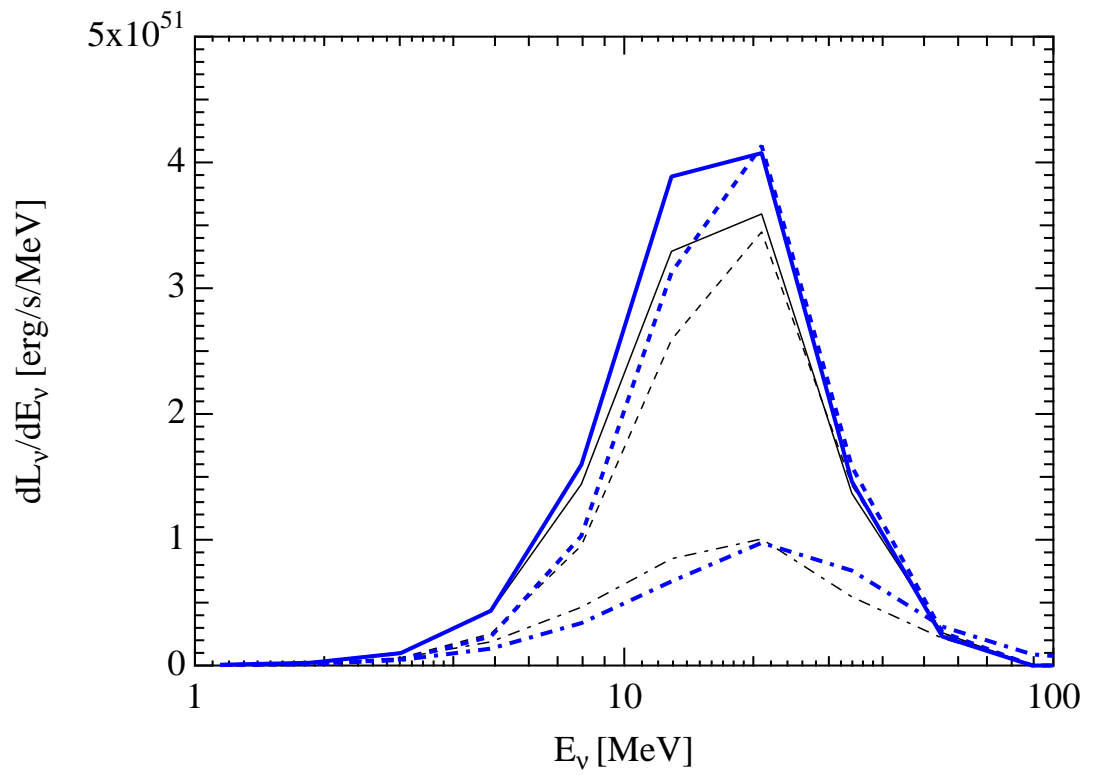

Fig. 11. - Energy spectra at $300 \mathrm{~ms}$ after bounce are shown as a function of neutrino energy for $\nu_{e}, \bar{\nu}_{e}$ and $\nu_{\mu / \tau}$ by solid, dashed and dash-dotted lines, respectively, for model H40L (thick) and W40L (thin). 